\title{
Increased nursing workload in patients with sepsis: A cohort study
}

\author{
Juliana AM Altafin ${ }^{1}$, Cintia MC Grion ${ }^{* 2}$, Ivanil AM Kauss ${ }^{1}$, Elza HT Anami ${ }^{1}$, Lucienne TQ Cardoso ${ }^{2}$, Fernanda \\ Chiqueti $^{2}$, Marjorie M Kaneshima ${ }^{2}$, Tiemi Matsuo ${ }^{3}$ \\ ${ }^{1}$ University Hospital, Londrina State University, Londrina, Brazil \\ ${ }^{2}$ Medical Department, Londrina State University, Londrina, Brazil \\ ${ }^{3}$ Statistics Department, Londrina State University, Londrina, Brazil
}

Received: October 15, 2015

Accepted: November 24, 2015 Online Published: December 2, 2015

DOI: $10.5430 /$ cns.v4n1p33

URL: http://dx.doi.org/10.5430/cns.v4n1p33

\begin{abstract}
Objective: To assess the nursing workload using the Nursing Activities Score (NAS) and compare results between patients with and without sepsis at an adult intensive care unit.

Methods: Prospective cohort study of patients admitted to the adult intensive care unit from March 10 to December 31, 2008. Clinical data of septic patients were collected in addition to data needed to calculate scores. Data were collected each day until release from the intensive care unit or up to 90 days of hospitalization. The nursing workload assessed using the Therapeutic Intervention Score System-28 and Nursing Activities Score relative to patients with and without sepsis were compared. The significance level was established at $5 \%$.

Results: A total of 437 patients were assessed (318 with and 119 without sepsis). The average nursing workload as measured by the Nursing Activities Score was high for the general intensive care unit (Nursing Activities Score $=74.7 \%$ ). Patients with sepsis were associated with greater nursing workloads during their first two weeks in the intensive care unit compared with patients without sepsis $(p<.05)$. Patients with septic shock were associated with greater nursing workloads (median Nursing Activities Score $=75.62 \%, p<.001$ ) compared with patients without sepsis (median Nursing Activities Score $=72.57 \%$ ), those with sepsis (median Nursing Activities Score $=70.20 \%$ ), and those with severe sepsis (median Nursing Activities Score $=72.65 \%$ ).

Conclusions: Sepsis increased nursing workload during the first two weeks after admission to the intensive care unit and the NAS may be useful to capture these differences.
\end{abstract}

Key Words: Organization and administration, Sepsis, Intensive care units, Nursing, Workload

\section{INTRODUCTION}

Intensive care units (ICUs) are the setting of care for patients with severe conditions. Thus, these units are characterized by great complexity, elevated costs, and the centralization of specialized technological resources. Factors such as technological advances, population aging, and the increasing numbers of chronic and critically ill patients currently influence the inpatient profile of ICUs, thereby leading to longer periods of hospitalization and greater degrees of care. ${ }^{[1]}$

The incidence of sepsis is high in ICU. ${ }^{[2]}$ Sepsis is a clinical condition associated with high mortality rates and complex treatments. A worldwide campaign was launched to establish recommendations to optimize the treatment of patients with sepsis and increase their chances of survival. ${ }^{[3]}$ These recommendations included nursing procedures and caregiving strategies in ICUs. Sixty-three nursing care rec-

\footnotetext{
* Correspondence: Cintia MC Grion; Email: cintiagrion@hotmail.com; Address: Departamento de Clinica Médica - CCS, Rua Robert Koch 60, Vila Operária, Londrina, Paraná, Brazil.
}

Published by Sciedu Press 
ommendations for patients with sepsis were described. ${ }^{[4]}$ The major recommendations focused on preventing nosocomial infections, early diagnosis leading to proper resuscitation measures, hemodynamic support, nutrition, eye and mouth hygiene, the prevention of pressure ulcers, and the management of nursing care.

These recommendations contributed to an increase in the nursing workload since they demand direct care of the patient on the bedside for longer periods of time. Frequently this increase in nursing workload meets an uneven ratio of nurses to the actual demand of nursing care. This lack of staffing interferes directly with patient safety, as well as the efficacy and quality of nursing. ${ }^{[5]}$ This scenario may create a problem of allocation of human resources, which led to the formulation of instruments to measure the demands of nursing care together with, or in parallel to, the creation of indices of severity and organ dysfunction for critically ill patients. ${ }^{[6]}$

In the last decade, the Nursing Activities Score (NAS) has been considered the most comprehensive measurement of nursing activity in adult ICUs, since it was designed to measure the actual average time it takes to perform each nursing activity. ${ }^{[7]}$ The application of the NAS not only aids the adequate allocation of human resources but also assesses the demand of nursing care and the everyday activities that require nurse participation. ${ }^{[8]}$

Due to the large effect that sepsis exerts on ICUs and the numerous recommendations related to the nursing care of patients with sepsis, studies are needed to assess whether sepsis influences the workload of ICU nurses. The present study assessed the nursing workload using the NAS and compared the results as they corresponded to patients with and without sepsis at an adult ICU.

\section{MethodS}

A prospective cohort study was conducted to evaluate nursing workload in patients admitted to the adult ICU of a University Hospital from March 10 to December 31, 2008. Patients were divided according to sepsis diagnosis to compare the results of nursing workload measured by NAS.

The sample was composed of all the consecutively admitted patients to the adult ICU throughout the study period. Patients younger than 18 years old and those who stayed in the ICU for less than 24 hours were excluded. In the case of patients who were eventually readmitted to the ICU during the study period, only the first admission was taken into consideration.

The following identification data were collected to characterize the patients: initials, gender, birthdate, clinic and medical 34 record numbers. The following ICU admission data were also collected: date and time of admission, origin (emergency room or hospital ward), reason for admission (medical, elective surgery, or emergency surgery), diagnosis at admission, date and time of ICU discharge, condition at ICU discharge (survivor or non-survivor), and condition at hospital discharge (survivor or non-survivor).

Data were also collected to calculate the NAS, as were the medical diagnosis records of sepsis and the data needed to calculate other scores with the purpose to characterize specific aspects of study population. Acute Physiology and Chronic Health Evaluation (APACHE II) ${ }^{[9]}$ was collected to describe severity of illness, Sequential Organ Failure Assessment (SOFA) ${ }^{[10]}$ to describe organ dysfunction, and Therapeutic Intervention Score System (TISS-28) ${ }^{[11]}$ to describe need for therapeutic interventions and as a complementary tool to measure nursing workload. APACHE II was collected from patient medical records at ICU admission and NAS, SOFA and TISS 28 were collected each day until their discharge from the ICU or up to 90 days of hospitalization.

The severity of sepsis was classified as sepsis, severe sepsis, or septic shock following the American College of Chest Physicians and the Society of Critical Care Medicine (ACCP/SCCM) consensus criteria. ${ }^{[12]}$ Although this consensus meeting has been held many years ago, definitions have not changed over this period. The present study considered patients diagnosed with sepsis, severe sepsis, or septic shock at any time during their stay in the ICU as septic. The remaining patients were considered non-septic.

NAS scoring was collected and calculated exclusively by one of the authors during the entire study period. This instrument is made up of eight major categories of care: monitoring and control; laboratory; medications; hygiene procedures; drain care; mobilization; support for the family and patient; administrative and managerial tasks (see Table 1).

To assess the nursing staff workload with NAS at the adult ICU the following features were considered:

(1) All 24-hour periods began and ended at 7:00 am.

(2) Nursing activities were recorded from the moment the patient was admitted to the ICU on the first day of hospitalization until the following day at 7:00 am.

(3) The record of interventions performed since the last NAS application to patient discharge from ICU was recorded.

(4) Each item was assigned a score (i.e., present) when the activity was performed on the assessed day and received the value equivalent to that activity. When a given activity was not performed, the score of the corresponding item was zero (i.e., absent).

ISSN 2324-7940 E-ISSN 2324-7959 
Table 1. Nursing Activities Score

\begin{tabular}{|c|c|}
\hline Activities & Score \\
\hline \multicolumn{2}{|l|}{ 1. Monitoring and control } \\
\hline Hourly vital signs, registration and calculation of fluid balance* & 4.5 \\
\hline Present at the bedside or active for two hours or more ${ }^{*}$ & 12.1 \\
\hline Present at the bedside or active for four hours or more ${ }^{*}$ & 19.6 \\
\hline 2. Laboratory investigations & 4.3 \\
\hline 3. Medications, except vasoactive drugs & 5.6 \\
\hline \multicolumn{2}{|l|}{ 4. Hygiene procedures } \\
\hline Performing hygiene procedures - normal ${ }^{*}$ & 4.1 \\
\hline Performing hygiene procedures - for two hours or more ${ }^{*}$ & 16.5 \\
\hline Performing hygiene procedures - for four hours or more* & 20.0 \\
\hline 5. Drain care, apart from nasogastric tubes & 1.8 \\
\hline \multicolumn{2}{|l|}{ 6. Mobilization and positioning } \\
\hline Performing procedures up to three times in 24 hours ${ }^{*}$ & 5.5 \\
\hline Performing procedures more frequently than three times in 24 hours or with two nurses with any frequency ${ }^{*}$ & 12.4 \\
\hline Performing procedures with three or more nurses with any frequency ${ }^{*}$ & 17.0 \\
\hline \multicolumn{2}{|l|}{ 7. Support and care for the family and patient } \\
\hline Support and care requiring full dedication for one hour ${ }^{*}$ & 4.0 \\
\hline Support and care requiring full dedication for one three hours or more* & 32.0 \\
\hline \multicolumn{2}{|l|}{ 8. Administrative and managerial tasks } \\
\hline Performing routine tasks* & 4.2 \\
\hline Performing tasks requiring full dedication for about 2 hours ${ }^{*}$ & 23.2 \\
\hline Performing tasks requiring full dedication for about 4 hours or more* & 30.0 \\
\hline 9. Ventilatory support & 1.4 \\
\hline 10. Care for artificial airways, orotracheal tube or tracheostomy cannula & 1.8 \\
\hline 11. Treatment for improving lung function & 4.4 \\
\hline 12. Vasoactive medication & 1.2 \\
\hline 13. Intravenous replacement of large fluid losses & 2.5 \\
\hline 14. Left atrium monitoring & 1.7 \\
\hline 15. Cardiopulmonary resuscitation in the previous 24 hours & 7.1 \\
\hline 16. Hemofiltration techniques, dialysis techniques & 7.7 \\
\hline 17. Quantitative urine output measurements & 7.0 \\
\hline 18. Measurement of intracranial pressure & 1.6 \\
\hline 19. Treatment of complicated metabolic acidosis or alkalosis & 1.3 \\
\hline 20. Intravenous hyperalimentation & 2.8 \\
\hline 21. Enteral feeding & 1.3 \\
\hline 22. Specific interventions in the intensive care unit & 2.8 \\
\hline 23. Specific interventions outside the intensive care unit & 1.9 \\
\hline
\end{tabular}

* = The sub-items of items 1, 4, 6, 7 and 8 are mutually exclusive

Local Human Research Ethics Committee approved this study and waived need for informed consent (Ruling no. 217/07) on October 24, 2007. This research was conducted in compliance with the required ethical standards.

After assessing whether the continuous quantitative variables were normally distributed using the Shapiro-Wilk test, they were expressed as the means and standard deviations or median and quartiles. The categorical variables were described as raw and relative frequencies. These results are presented in tables and charts.
The categorical variables were compared using Pearson's chisquared test. To compare two groups of continuous variables, Student's independent-samples $t$-test was used for normally distributed variables, and the Mann-Whitney U test was used for variables with non-normal distributions. To compare continuous, non-normally distributed variables with three or more levels, homogeneous variances, or both, the KruskalWallis test was applied. Dunn's post hoc tests were used to determine which groups specifically differed.

The significance level was established as $5 \%$, and confidence 
intervals were set at 95\%. Data were entered on Epi Info (version 3.3.2, 2005, CDC, USA) and statistical analysis were performed on SAS (version 8.2, SAS Institute, Cary, NC, USA).

\section{Results}

A total of 622 patients were admitted to the ICU over the study period, 185 patients met exclusion criteria. Therefore, 437 patients were assessed; 318 had sepsis, and 119 did not have sepsis (see Figure 1).

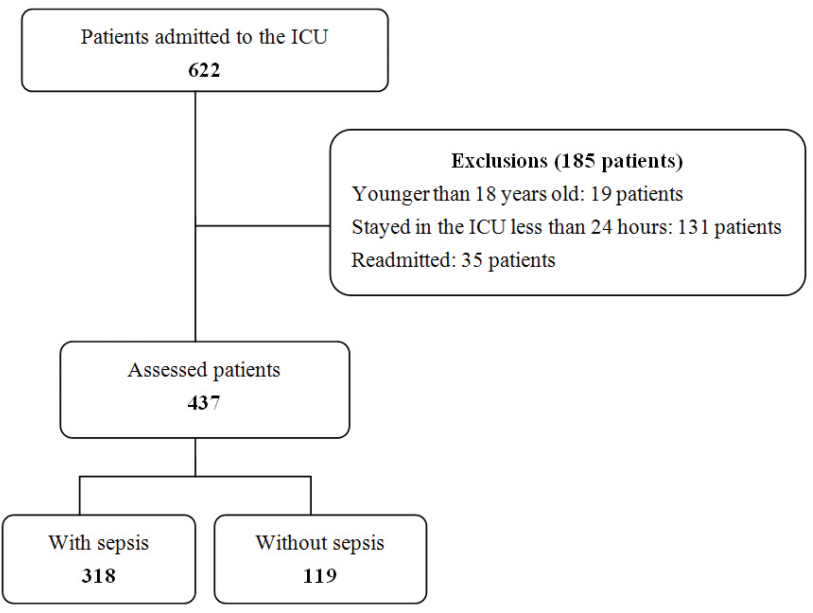

Figure 1. Flowchart of the patients admitted to the adult Intensive Care Unit

The majority of patients were male (57.0\%), and the average age was $58.2(\mathrm{DP}=18.9)$ years old. The most frequent medical diagnoses at admission were sepsis $(51.5 \%)$, post-cardiac arrest syndrome (6.6\%), and intracranial hemorrhage (4.3\%). The most frequent surgical reasons for admission to the ICU were postoperative care with regard to brain $(9.7 \%)$ chest $(5.5 \%)$, heart $(5.2 \%)$, or peripheral vascular $(3.0 \%)$ surgery.

The average APACHE II score was 21.7 (DP = 9.51). According to APACHE II diagnostic categories, $12.1 \%$ of these patients were diagnosed with chronic disease: $5.5 \%$ were diagnosed with immunodeficiency, $3.2 \%$ were diagnosed with liver cirrhosis, $1.6 \%$ were diagnosed with chronic obstructive pulmonary disease, $1.1 \%$ were diagnosed with heart failure, and $0.7 \%$ were diagnosed with chronic kidney failure.

Sepsis, severe sepsis, or septic shock was diagnosed in $318 / 437$ (72.8\%) patients at some point during their stay at the ICU. Approximately half of the patients (49.4\%) stayed 3 to 10 days at the ICU, with a median of 5 days. The adult ICU mortality rate was $41.0 \%$, and the in-hospital mortality rate was $49.2 \%$.

The primary infection of the 318 septic patients was pulmonary $(81.7 \%)$, followed by urinary $(20.7 \%)$, abdominal
$(13.8 \%)$, skin and soft tissue (6.9\%), indeterminate (5.9\%), blood $(3.1 \%)$, other $(2.8 \%)$, bones $(1.9 \%)$, surgical site $(0.6 \%)$, and catheter $(0.3 \%)$ infections. Fifty patients exhibited more than one type of sepsis, of which pulmonary and urinary infections were the most frequent $(n=19)$.

Patient clinical and demographic characteristic comparisons by the occurrence of sepsis are described in Table 2. As established by the average TISS-28 score during the full stay at the ICU, the nursing workload was higher when associated with septic patients compared with non-septic patients $(p<$ .001). However, this difference was not significant when the nursing workload was assessed using median NAS during the entire ICU stay $(p=.085)$.

The median NAS was higher in septic than in non-septic patients during their first two weeks of treatment at the ICU (see Figure 2). With regard to workload by sepsis severity, the median NAS was higher during the stay at the ICU among patients with septic shock than the remainder of the sample (see Table 3).

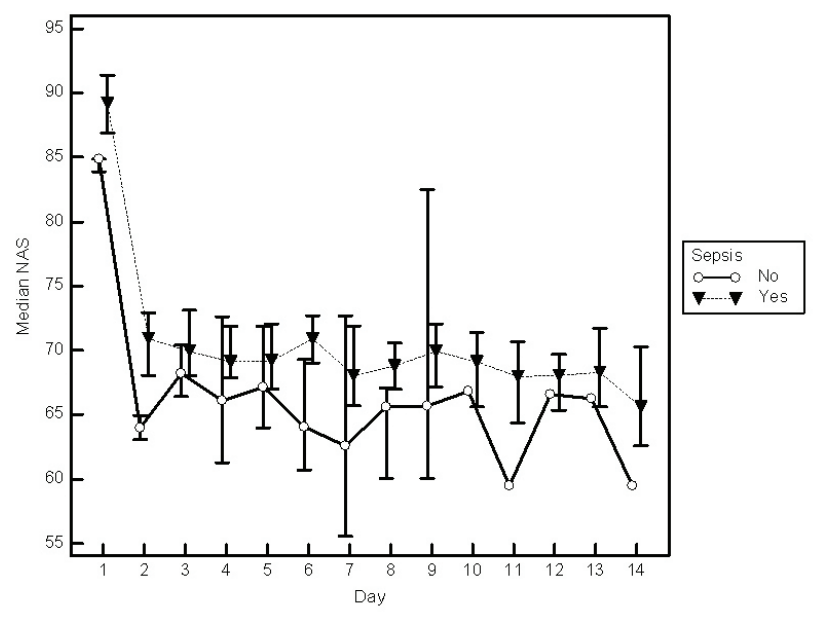

Figure 2. A comparison of the nursing workload for patients with and without sepsis as assessed by the daily median NAS

NAS: Nursing Activities Score; $p<.05$ for each day:

Mann-Whitney U test

To identify the types of nursing activities most frequently associated with sepsis, frequency of septic patients among those requiring each NAS activity was calculated at ICU admission. At admission to the ICU, 225 patients had sepsis, and 11 of the 32 nursing activities measured by NAS were more likely to be required when a high frequency of septic patients existed, these activities are listed as follows: (1) Presence at bedside and continuous observation or activity for 4 hours or more within any shift for reasons of safety, severity, or treatment; (2) Care of drainage; (3) Any 
modality of mechanical/assisted ventilation including spontaneous respiration, with or without positive end-expiratory pressure or with or without endotracheal tube and any method of supplemental oxygen administration; (4) Care of artificial airways; (5) Chest physical therapy, incentive spirometry, inhalation therapy, or endotracheal aspiration; (6) Va- soactive medication; (7) Intravenous replacement of large fluid volumes; (8) Hemofiltration or dialysis techniques; (9) The treatment of complicated metabolic acidosis/alkalosis; (10) Enteral feeding; (11) Endotracheal intubation, pacemaker insertion, cardioversion, endoscopy, emergency surgery over the past 24 hours, and gastric lavage.

Table 2. Comparison of clinical and demographic characteristics between patients with and without sepsis

\begin{tabular}{|c|c|c|c|}
\hline \multirow{2}{*}{ Variables } & \multicolumn{2}{|c|}{ SEPSIS } & \multirow{2}{*}{$p$-value } \\
\hline & Yes $(n=318)$ & No $(n=119)$ & \\
\hline Gender male (n, \%) & $193(77.5 \%)$ & $56(22.5 \%)$ & $.010^{*}$ \\
\hline \multicolumn{4}{|l|}{ Age (years; n, \%) } \\
\hline $18-40$ & 65 (75.6\%) & $21(24.4 \%)$ & $<.001^{*}$ \\
\hline $41-59$ & $70(56.5 \%)$ & $54(43.5 \%)$ & \\
\hline $60-79$ & $137(78.7 \%)$ & 37 (21.3\%) & \\
\hline $80-100$ & $46(86.8 \%)$ & $7(13.2 \%)$ & \\
\hline Provenance Emergency room (n, \%) & $196(88.3 \%)$ & $26(11.7 \%)$ & $<.001^{*}$ \\
\hline \multicolumn{4}{|l|}{ Hospitalization type (n, \%) } \\
\hline Clinical & $218(87.6 \%)$ & $31(12.4 \%)$ & $<.001^{*}$ \\
\hline Elective surgery & $44(37.3 \%)$ & $74(62.7 \%)$ & \\
\hline Emergency surgery & $56(80.0 \%)$ & $14(20.0 \%)$ & \\
\hline APACHE-II score (Mean, SD) & $24.7(9.0)$ & $13.7(5,3)$ & $<.001^{* *}$ \\
\hline Average SOFA (Mean, SD) & $8.3(4.2)$ & $3.17(2.3)$ & $<.001^{* *}$ \\
\hline Average TISS-28 (Mean, SD) & $27.78(6.1)$ & $20.45(4.9)$ & $<.001^{* *}$ \\
\hline Average NAS (Mean, SD) & $75.14(9.2)$ & $72.69(7.4)$ & $.085^{* *}$ \\
\hline Length of stay at the ICU (Mean, SD) & $11.62(13.6)$ & $2.64(1.4)$ & $<.001^{* *}$ \\
\hline ICU mortality (n, \%) & $174(97.2 \%)$ & $5(2.8 \%)$ & $<.001^{*}$ \\
\hline In-hospital mortality (n, \%) & 203 (94.4\%) & $12(5.6 \%)$ & $<.001^{*}$ \\
\hline
\end{tabular}

Note. ${ }^{*}$ Chi-squared test; ${ }^{* *}$ Student's t-test; APACHE II: Acute Physiology and Chronic Health Evaluation; SD: standard deviation; NAS: Nursing Activities Score; SOFA: Sequential Organ Failure Assessment; TISS-28: Therapeutic Intervention Scoring System; ICU: intensive care unit

\section{Discussion}

The present study investigated the nursing workload using the NAS as it related to patients admitted to a medical-surgical ICU. The mean NAS corresponding to patients with and without sepsis was compared; we found that the nursing workload was significantly higher among septic patients during their first week of stay at the ICU.

The findings of the present study has implications to clinical practice since patient safety and quality of nursing care can be negatively affected by reduced staffing. Nurses' work should be carefully planned for ICU with high incidence of sepsis diagnosis according to priority setting, delegation, interactions and collaborations. Furthermore, reallocation of human resources should be considered to meet the nursing work's demand and ensure an adequate patient to nurse ratio. Improvement of healthcare quality and safety implies in the ability to view how one component of the work system is related to other components and to the whole. ${ }^{[13]}$ Since data acquisition and results of the present study, staffing needs and demands have improved in the local institution of research. An institutional protocol was implemented and a Sepsis Team has been created to offer support and orientation for the initial steps of sepsis treatment, as well as additional human resources were allocated in the ICU.

The demographic and clinical characteristics of the $437 \mathrm{pa}-$ tients admitted to the adult ICU who comprised the sample were similar to those reported by others studies conducted with severely ill patients. ${ }^{[10,14,15]}$ The average APACHE II score was high compared with other studies using this index. ${ }^{[15,16]}$ Importantly, however, the average APACHE II score was higher among studies conducted with septic patients. ${ }^{[17,18]}$ The degree of patient severity in the present study was measured by the high SOFA scores, which reflected the presence of organ dysfunction and failure. 
Table 3. Comparisons of NAS among patients without sepsis and those with sepsis, severe sepsis, or septic shock

\begin{tabular}{lllll}
\hline Sepsis & N & NAS median & Interquartile deviation & Comparisons $^{*}$ \\
\hline Without sepsis & 119 & 72.57 & 6.87 & $\mathrm{~A}$ \\
Sepsis & 75 & 70.20 & 6.52 & $\mathrm{~A}$ \\
Severe sepsis & 83 & 72.65 & 10.82 & $\mathrm{~A}$ \\
Septic shock & 160 & 75.62 & 11.78 & $\mathrm{~B}$ \\
\hline
\end{tabular}

Note. NAS: Nursing Activities Score; Kruskal-Wallis: $\mathrm{H}=27.84$ with 3 degrees of freedom and $p$-value $<.001$; ${ }^{*}$ Groups with the same letter did not exhibit significant difference on a Dunn's post hoc test

The mean NAS was high in the present sample compared with other studies. ${ }^{[19,20]}$ Previous study reported mean NAS similar to ours in a cardiac postoperative care unit, indicating the greater complexity of the medical needs of these patients and the resulting high demand of nursing care. ${ }^{[21]}$ Thus, we highlight the importance of these data relative to the allocation of nursing resources.

According to our findings, sepsis was the primary reason for admission to the ICU, and a large proportion of patients developed sepsis during their stay at the ICU. These findings have been already described and indicate the importance of studying sepsis diagnoses and their effect on nursing activities in the ICU. ${ }^{[22]}$ The increased workload during the first week most likely reflects the application of the early care measures recommended by the international guidelines for patients with sepsis and the measures taken to prevent and treat the organ dysfunctions that commonly occur among these patients at the onset of treatment. ${ }^{[3]}$

Recent study found that excessive nursing workload was the most important risk factor for acquiring nosocomial infection. ${ }^{[23]}$ Previous studies have found associations between higher nursing workloads and patients with fevers or infections. ${ }^{[24-26]}$ These studies typically applied the TISS-28 to assess the workload, and the average scores were higher for those who nursed patients with ventilator-associated pneumonia and bloodstream infections. Interestingly, our study also revealed that the average TISS- 28 score identified an increase in the nursing workload across the full ICU stay of patients with sepsis compared with those without sepsis; however, the NAS did not confirm this finding. The sample size might not have been large enough to detect this difference throughout the ICU stay. Nevertheless, this result was found during the first week following admission when treatment is typically more intense, especially among patients with satisfactory clinical progression because the nursing workload tends to decrease as the time of discharge from the ICU nears.

Regarding the relative usefulness of NAS and TISS 28 when measuring nursing workload, it has been described that TISS 28 underestimates ICU nursing activities compared to NAS. For TISS 28, the time associated with each point was at- tributed by a panel of experts, while NAS' points measure the actual average time it takes to perform each nursing activity, by using a statistical methodology named "work-sampling" technique. ${ }^{[7]}$ The selection of items for NAS score describes nursing activities and not patient conditions or technologic interventions which do not necessary correspond to an increase in the consumption of nursing work, therefore NAS applies exclusively to activities performed by professionals included in the description of the nursing staff in the ICU.

After dividing patients by the severity of sepsis, those with the most severe levels of organic dysfunction (i.e., those with septic shock) demanded a greater workload from the nursing staff compared with the remaining patients. Patients with septic shock might demand a greater workload from nurses because the severity of their condition demands more nursing procedures and a closer monitoring of their clinical progression, thereby resulting in an increased presence of nursing staff at patients' bedsides.

With regard to septic patients at admission to ICU, the nursing activities assessed by NAS most frequently performed were those that required a continuous presence of nurses at the bedside due to the use of monitoring devices and interventions to treat organ dysfunctions. A study conducted in Greece compared the nursing workload associated with 361 patients with or without fever as measured by the TISS- 28 and found that five categories were significantly associated with patients with (but not those without) fever: basic activities, ventilator support, metabolic support, neurological support, and specific interventions. ${ }^{[27]}$ Similar to our study, the NAS and TISS-28 categories measure increases in the nursing workload.

The limitations of the present study should be considered. Because it was conducted at one center, it may be considered as a pilot study and generalizations of our results require caution. Our sample size did not allow us to establish whether any differences in the nursing workload existed over the full period of stay at the ICU for septic patients in the same way that it was determined for the first week. Multicenter studies with larger samples might provide more consistent results in this regard. 
In conclusion, the present study showed that nursing workload seems to be increased in patients with sepsis during the first two weeks after admission to the intensive care unit and the Nursing Activities Score may be useful to capture these differences. Patients with septic shock were associated with the greatest nursing workload.

\section{REFERENCES}

[1] Amaral AC, Rubenfeld GD. The future of critical care. Curr Opin Crit Care. 2009; 15: 308-13. http://dx.doi.org/10.1097/MCC .0b013e32832e4550

[2] Cawcutt KA, Peters SG. Severe sepsis and septic shock: clinical overview and update on management. Mayo Clin Proc. 2014; 89(11): 1572-8. http://dx.doi.org/10.1016/j.mayocp. 2014.07.00 9

[3] Dellinger RP, Levy MM, Rhodes A, et al. Surviving Sepsis Campaign Guidelines Committee including the Pediatric Subgroup. (2013). Surviving Sepsis Campaign: International guidelines for the management of severe sepsis and septic shock. Crit Care Med. 2012; 39(2): 165228. http://dx.doi .org/10.1097/CCM. Ob013e31827e83af

[4] Aitken LM, Williams G, Harvey M, et al. Nursing considerations to complement the Surviving Sepsis Campaign guidelines. Crit Care Med. 2011; 39(7): 1800-18. http://dx.doi.org/10.1097/CCM $.0 \mathrm{~b} 013 \mathrm{e} 31821867 \mathrm{cc}$

[5] Padilha KG, Sousa RMC, Queijo AF, et al. Nursing Activities Score in the intensive care unit: analysis of the related factors. Intensive Crit Care Nurs. 2008; 24(3): 197-204. PMID:17976989. http://dx.doi.org/10.1016/j.iccn.2007.09.004

[6] Gonçalves LA, Padilha KG. Fatores associados à carga de trabalho de enfermagem em Unidade de Terapia Intensiva [Factors associated with nursing workload in adult intensive care units]. Rev Esc Enferm USP. 2007; 41(4): 645-52. PMID:18193620. http: //dx.doi.org/10.1590/S0080-62342007000400015

[7] Miranda DR, Jegers M. Monitoring costs in the ICU: a search for a pertinent methodology. Acta Anaesthesiol Scand. 2012; 56(9): 110413. http://dx.doi.org/10.1111/j.1399-6576.2012.02735 $\mathrm{x}$

[8] Padilha KG, Sousa RMC, Garcia PC, et al. Nursing workload and staff allocation in an intensive care unit: a pilot study according to Nursing Activities Score (NAS). Intensive Crit Care Nurs. 2010; 26(2): 108-13. http://dx.doi.org/10.1016/j.iccn.2009.1 2.002

[9] Lee H, Lim CW, Hong HP, et al. Efficacy of the APACHE II score at ICU discharge in predicting post-ICU mortality and ICU readmission in critically ill surgical patients. Anaesth Intensive Care. 2015 Mar; 43(2): 175-86. PMID:25735682.

[10] Anami EHT, Grion CMC, Cardoso LTQ, et al. Serial evaluation of the SOFA score in a Brazilian teaching hospital. Intensive Crit Care Nurs. 2010; 26(2): 75-82. http://dx.doi.org/10.1016/j.icc n. 2009.10.005

[11] De Souza Urbanetto J, Travi Canabarro S, Prado Lima Figueiredo AE, et al. Correlation between the TISS-28 and NEMS indicators in an intensive care unit. Int J Nurs Pract. 2014 Aug; 20(4): 375-81. http://dx.doi.org/10.1111/ijn.12183

[12] Levy MM, Fink MP, Marshall JC, et al. International Sepsis Definitions Conference. Crit Care Med. 2003; 31(4): 1250-6. PMID:12682500. http://dx.doi.org/10.1097/01.CCM.0000 $050454.01978 .3 \mathrm{~B}$

[13] Dolansky MA, Moore SM. Quality and safety education for nurses (QSEN): The key is system thinking. OJIN. 2013; 18(3): Manuscript 1.

Published by Sciedu Press
[14] Cudak EK, Dyk D. Nursing demand in intensive therapy units assessed by the Nursing Activities Score. Anestezjol Intens Ter. 2010; XLII(2): 62-6.

[15] Moran JL, Bristow P, Solomon PJ, et al. The Australian and New Zealand Intensive Care Society. Mortality and length-of-stay outcomes, 1993-2003, in the binational Australian and New Zealand intensive care adult patient database. Crit Care Med. 2008; 36(1): 46-61. PMID:18090383. http://dx . doi .org/10.1097/01 . CCM. 0000295313.08084 .58

[16] Kiekkas P, Brokalaki H, Manolis E, et al. Patient severity as an indicator of the nursing workload in the intensive care unit. Nurs Crit Care. 2007; 12(1): 34-41. PMID:17883662. http://dx.doi.org /10.1111/j.1478-5153.2006.00193.x

[17] Zhou J, Qian C, Zhao M, et al. China Critical Care Clinical Trials Group. Epidemiology and outcome of severe sepsis and septic shock in intensive care units in mainland China. PLoS One. 2014; 9(9): e107181. http://dx.doi.org/10.1371/journal.pone 0107181

[18] De Carvalho MA, Freitas FG, Silva Junior HT, et al. Mortality predictors in renal transplant recipients with severe sepsis and septic shock. PLoS One. 2014 Nov 4; 9(11): e111610. http: //dx.doi.o rg/10.1371/journal. pone.0111610

[19] Queijo AF, Martins RS, Andolhe R, et al. Nursing workload in neurological intensive care units: cross-sectional study. Intensive Crit Care Nurs. 2013; 29(2): 112-6. http://dx.doi.org/10.1016/j .iccn.2012.08.001

[20] Carmona-Monge FJ, Rodríguez GMR, Herranz CQ, et al. Evaluation of the nursing workload through the nine equivalents for nursing manpower use scale and the nursing activities score: a prospective correlation study. Intensive Crit Care Nurs. 2013; 29(4): 228- 33 http://dx.doi.org/10.1016/j.iccn.2013.03.003

[21] Ducci AJ, Zanei SSV, Whitaker IY. Nursing workload to verify nurse/patient ratio in a cardiology ICU. Rev Esc Enferm USP. 2008; 42(4): 673-80. PMID:19192901. http://dx.doi.org/10.1590 /S0080-62342008000400009

[22] Kauss IA, Grion CM, Cardoso LT, et al. The epidemiology of sepsis in a Brazilian teaching hospital. Braz J Infect Dis. 2010; 14(3): 264 70. http://dx.doi.org/10.1016/S1413-8670(10)70054-4

[23] Daud-Gallotti RM, Costa SF, Guimarães T, et al. Nursing workload as a risk factor for healthcare associated infections in ICU: a prospective study. PLoS One. 2012; 7(12): e52342. http://dx.doi.org /10.1371/journal. pone.0052342

[24] Saulnier FF, Hubert H, Onimus TM, et al. Assessing excess nurse work load generated by multi-resistant nosocomial bacteria in intensive care. Infect Control Hosp Epidemiol. 2001; 22(5): 273-8. PMID:11428436. http://dx.doi.org/10.1086/501899

[25] Apostolopoulou E, Veldekis D. Excess nursing workload and extra cost attributable to ventilator-associated pneumonia in intensive care units. ICUS Nurs Web J. 2004; 20: 1-8.

[26] Apostolopoulou E, Veldekis D. Nosocomial bloodstream infections in intensive care units: Excess nursing workload, extra costs. ICUS Nurs Web J. 2005; 24: 1-5.

[27] Kiekkas P, Sakellaropoulos GC, Brokalaki H, et al. Nursing workload associated with fever in the general intensive care unit. Am J Crit Care. 2008; 17(6): 522-31. PMID:18978237. 